\title{
MULTI-AGENT TASKS SCHEDULING FOR COORDINATED ACTIONS OF UNMANNED AERIAL VEHICLES ACTING IN GROUP
}

\author{
PETR SKOBELEV ${ }^{1}$, DENIS BUDAEV ${ }^{2}$, ALEKSEY BRANKOVSKY ${ }^{2} \&$ GEORGY VOSCHUK $^{2}$ \\ ${ }^{1}$ Samara National Research University, Samara State Technical University, Samara, Russia. \\ ${ }^{2}$ Smart Solutions, Ltd, Samara, Russia.
}

\begin{abstract}
This paper discusses hardware and software prototype of multi-agent system for unmanned aerial vehicles (UAVs) group action planning. We describe the approach for system implementation as a whole and software agents within the system. The aim of current and future developments is creation of complex scientific and technical solutions for coordinated planning and actions management of heterogeneous UAV groups in real time.

Keywords: adaptability, coordinated control, drones, dynamic rescheduling, intelligence, multi-agent systems, real time, UAV, Unmanned aerial vehicle
\end{abstract}

\section{INTRODUCTION}

The use of classical optimization methods is not always effective for complex multifactorial problems, especially with high dynamics of events affecting the results of the planning. Reconciliation in a distributed computing environment also requires special approaches. An example of such complex problems is coordinated management of a group of unmanned aerial vehicles (UAVs) in real time.

Unmanned aerial vehicles (UAVs or drones) are widely used in various sectors of economy. Main advantages of using drones are achieved when used in critical missions that require response in the shortest possible time. For example, UAVs can search for survivors at emergency scenes, which provides quick access to areas where human access is impossible or dangerous. However, to ensure the implementation of the assigned tasks in the shortest possible time and improve the quality authors consider using several drones acting together as a coordinated group. Such a group of drones with a joint goal should coordinate and reconcile mutual tasks. As a result, a group of UAVs acting together will be able to perform tasks faster.

However, there is an issue with planning tools for real-time control of UAVs groups, especially in unforeseen circumstances. For example, there is lack of software, which can manage UAVs group in case of failure of a UAV, addition of a new area, or addition of a new UAV in a group. Most of the existing software solutions are designed for planning one autonomous UAV. On the other hand, drone group management software would use available UAVs resources in the most efficient way (battery or fuel supplies, time resources, hardware/computing resources of UAVs). In addition, quick distribution of tasks between the drones during mission execution would reduce total time.

For effective management of resource allocation (incl. drones and their subsystems) it is expedient to use scheduling systems. Nowadays these kinds of systems use the following methods of complex problem solving:

- traditional methods of optimization and linear programming in the area of mixed realvalued, integral-valued and logical variables, the improvement of precise methods of tasks solving, such as 'branch and bound' methods, nonlinear programming methods, methods of constraint programming [1]; 
- greedy algorithms, based on heuristic business-rules for specific subject areas;

- artificial intelligence methods, the use of neural networks and fuzzy logic;

- metaheuristics (local search, Tabu Search, GRASP algorithms) [2];

- bio-inspired methods: Ant Colony Optimization (ACO), Artificial Bee Colony (ABC), Bio Inspired and a similar methods, as well Simulated Annealing (SA), Monte-Carlo method and some others [3].

Many scheduling systems are based on centralized and deterministic principles. However, distributed coordination in networks of dynamic agents has attracted an interest of numerous researchers in recent years. As shown in Refs. $[4,5]$, the multi-agent technology methods are the most promising and appropriate for the resources allocation algorithm design. The following paper describes the practical experience of using multi-agent technology methods for developing a system for UAVs group management. We describe task formulation for terrain survey with UAVs group and decentralized multi-agent, multi-criteria task scheduling solution process. We also describe the results of the software and hardware implementation of the system along with some experimental and real flights results.

\section{PROBLEM STATEMENT}

Let us consider problem formulation with the following initial data:

- area for survey mission that is limited in size and may be identified by boundary points coordinates;

- topography information about the area (terrain heights);

- collection of available UAVs, which may be included in a group;

- technical specification of UAVs and equipment - ranges of available speeds and altitudes, battery capacity, charging time, camera information.

One of the main features of proposed UAVs group management approach is the ability of UAVs to communicate over the wireless network. In addition, each UAV can fly in standalone mode along the path trajectory identified by checkpoints, and each unit can 'coordinate' actions with other devices by sending and receiving data messages via wireless communication channels.

\section{UAV GROUP MANAGEMENT}

UAVs have expanded the scope of their application in recent years quite extensively. For example, rescue services use UAVs for forests monitoring, fires search. UAVs re successfully used in the search and rescue missions. UAVs can help solve problems in the areas with difficult or dangerous access for humans.

Using UAVs increases the effectiveness of rescue operations because they are mobile and require less time to prepare for the flight and launch (when compared to the large aircrafts). UAVs are indispensable for creation of accurate digital models of territories with a dense arrangement of objects and buildings.

Most of the existing software solutions are designed for planning and management activities of alone acting UAV. However, some missions require rapid tasks distribution among available drones. It is critical to calculate individual routes and actions of each UAV and reconcile resulting routes especially in high dynamics of events affecting the results. 
Ordinary methods are not always effective for solving complex multifactorial problems, especially in high dynamics in distributed environment. One approach to solving the problem of resource management involves the use of multi-agent technology (MAT).

As part of the multi-agent approach, each active entity type within a solved problem is represented by a software 'agent', formalizing the logic and needs of the entity. All UAVs have on board a separate single-board computer with MAT software, so UAVs can share information wirelessly. Scheduling in this case is a process of negotiation between these agents in order to determine the compromise resulting plan.

For UAV group management we introduce the concept of observation square. The surveying area is divided into a finite amount of observation squares according to characteristics of the UAVs and their equipment. For example, for the UAV with camera sensor width $4.55 \mathrm{~mm}$, the focal length $3.61 \mathrm{~mm}$, the flight height $119 \mathrm{~m}$ (FAA restricts flights for small UAVs above 400 feet altitude), the image width and height 4000 and 3000 pixel, respectively, we expect ground sampling distance (GSD) of $3.75 \mathrm{~cm} / \mathrm{pixel}$ and image footprint on the ground $150 \mathrm{~m}$. Therefore, for the area of $32 \times 32$ observation squares we expect the total area of 23.04 square kilometers. Thus, there is a survey mission with 1024 sub-tasks for available amount of UAVs.

Each observation square is associated with its sub-task to perform. Each sub-task has a timestamp of UAV flight and its format is hh:mm:ss. The timestamp is renewed at the next UAV flight over the observation square. Considering these facts, UAVs agents can track how long the square was without supervision. It is important for the patrolling mission planning.

After operator sets mission parameters (sets the boundary points of observation area, selects a mission type, for example, 'single flight over area' and determines the UAVs in-group), all sub-tasks become available for scheduling and are transmitted to UAVs matching mechanism. This mechanism is responsible for the initial distribution of individual sub-tasks (observation squares) between individual UAVs of group. In fact, the mechanism realizes clustering to aggregate observations squares.

After the initial clustering each UAV agent receives its individual area within the corresponding cluster, that is, some set of observations squares, in which the agent has to build a flight path to perform UAV area supervision.

The process of flight path calculation is performed by UAV agent. UAV agent analyzes the list of available sub-tasks and evaluates alternatives sets of paths based on the set of planning criteria. Final list of planning criteria is primarily determined by the type of the mission.

Authors consider that it is important to build such multi-agent scheduling algorithms that UAVs have the least possible number of turns and backward turns. To solve this issue by analogy with the method proposed in [6] for generating a path for a single UAV, we propose an algorithm modification for UAVs group.

During the planning process and performance of in-flight sub-tasks each UAV can reallocate sub-tasks according to agent key performance indicators. This approach allows controlling sub-tasks distribution and balancing in real time, and unlike similar methods of path forming [6-9], it is originally designed to increase total system performance, resources utilization and reduce execution time. Agent key performance indicators (KPI) are applied to evaluate and compare different options of sub-tasks distribution based on criteria of UAV agent satisfaction. For prototype system two criteria have been chosen to calculate the agent satisfaction: 
1. Criterion of area observability. This criterion depends on sub-tasks squares observability. The meaning of this criterion is to enforce agents selecting those observation squares which have been most unexplored in the previous time intervals. UAV agent satisfaction depends on the time during which the square remained without supervision, that is, from the time during which no UAVs of a group were flying over a square for observing.

2. Criterion of total UAVs path distance. The meaning of the criterion is to enforce agents selecting a set of squares which lies on the same line. This provides efficient use of UAVs resources - minimum number of turns on the path and minimum distance without observing a square. The criterion defines satisfaction of UAVs agents from alternatives of observation squares selecting. UAVs agent KPI are higher when his path contains a set of observations squares lying on a straight line. The attractiveness of selected squares set for UAVs agent is higher, the higher the ratio of paths length on observed squares to the total path length.

The overall system satisfaction at a certain stage of work is defined as the sum of all UAVs agents satisfaction on the criterion of area observability and on the criterion of total UAVs path distance considering criteria weights (sum of the weights is always equal to 1).

$$
K P I_{\text {system }}=k_{\text {area observ. }} * \sum_{i=1}^{N} K P I_{i \text { area observ. }}+k_{\text {path dist. }} \sum_{i=1}^{N} K P I_{i \text { path dist. }}
$$

During coordination of the flight plan in the group, each UAV provides a set of possible options for changing position. Each of these options is characterized by satisfaction indicators for UAV (observability and path distance). The overall system satisfaction at a particular planning stage is defined as the sum of KPIs of all agents, considering criteria weights. The higher the total KPI of the system, the better the quality of solution in the context of the selected criteria.

Thus, the total flight plan is a set of options for all devices of a group, one approved option from the list of alternative options for each UAV, which maximizes KPI of the system.

Realized dynamic scheduling mechanism provides adaptive relocating of individual observation squares between UAVs in the group to minimize difference between completion times between different UAV devices.

A process of sub-squares relocating between two UAVs is performed as follows:

1. During flights UAVs exchange data about execution time forecasts.

2. UAV detects a significant difference (parameter) between its completion time forecast and the time of another UAV.

3. UAV agent calculates how many sub-tasks to transfer from or to other UAV to reduce the difference between completion times.

4. UAV agent sends a request to reallocate the calculated number of sub-tasks.

5. Sub-tasks are reallocated or the agent receives a rejection (for example, if UAV has already transferred some of the squares to another third UAV).

Thus, adaptive balancing mechanism ensures that the mission is performed in the shortest time even in cases of UAVs various performance and unplanned events. 


\section{RESULTS}

Authors have completed a series of experiments to research the system performance. The input data contained survey area, collection of available UAVs (software-hardware simulations with PX4 flight controller boards). The total amount of observation squares was 1024 . The main purpose of experiments was to evaluate effectiveness of sub-tasks distribution by UAVs of the group, assess formed UAV flight plan, estimate time required for planning and preparing flight plans, and assess systems reaction to tasks changing during planning. During tests, we launched scheduling process for different amount of UAVs in group and measured parameters of system performance. Results of experiments are shown in Table 1.

- Firstly, total time spent on tasks performing directly depends on the number of observation squares in the flight mission and the number of UAVs devices involved in the planning process. With the same number of squares the more devices are involved in the planning process, the faster the system can issue a total flight plan. This is explained by the distributed nature of planning mechanism, which allows use of all resources of computing devices in the planning process, which reduces the time for preparing the final total flight plan. Scheduling for a single-working UAV may take longer than for united group of devices, since all subtasks have to be consistently rated by one device.

- Secondly, the greater the number of UAVs involved in mission, the less time is required to perform all tasks of the total flight plan. This is easily explained by the fact that each of UAVs works in its responsibility area concomitantly coordinating work plans and levelling load with all UAVs from a group using the load balancing mechanism that reduces the total time of full mission execution.

- Thirdly, the system reaction time to changes in environment is less when a smaller number of UAVs is affected by this change, because coordinating all changes requires negotiation between all agents affected by these changes.

Also overall efficiency of the system is characterized by the indicator of total KPI of the system which in turn is determined by satisfaction level of each UAV agent. Generally, the KPI value for a single-working UAV will be higher than the overall KPI value for a group of coordinated devices which are forced to compete and negotiate, by compromising. In other words, KPI index is only a signal about necessity of plan optimization during system performance and these indicators only can be compared with similar indicators of similar UAVs groups, for example, similar UAVs types and number of devices.

Table 1: Summary results of experiments.

\begin{tabular}{|c|c|c|c|c|}
\hline Indicator & $\begin{array}{l}\text { Group of } \\
3 \text { UAVs }\end{array}$ & $\begin{array}{l}\text { Group of } \\
4 \text { UAVs }\end{array}$ & $\begin{array}{l}\text { Group of } \\
10 \text { UAVs }\end{array}$ & $\begin{array}{l}\text { Single } \\
\text { UAV }\end{array}$ \\
\hline Time for full mission planning, seconds & 62 & 50 & 21 & 165 \\
\hline $\begin{array}{l}\text { Forecasted time for mission performance, } \\
\text { minutes }\end{array}$ & 404.23 & 304.23 & 145.33 & 1205.13 \\
\hline $\begin{array}{l}\text { Reaction time to tasks addition (time of } \\
\text { sub-tasks relocation between UAVs), } \\
\text { seconds }\end{array}$ & 9 & 15 & 13 & 6 \\
\hline $\begin{array}{l}\text { Overall system KPI (satisfaction of all } \\
\text { agents) after planning, } \%\end{array}$ & 78 & 79 & 68 & 95 \\
\hline
\end{tabular}


Multi-agent planning methods provide the ability to manage a group of UAVs and monitor and adjust performance of the group through agent key performance indicators and criteria.

\section{CONCLUSION}

Software systems for management and control of robotic devices including united UAVs groups are actively developed at the present time [10, 11]. A promising direction for developing such systems is extending their functionality by developing technical solutions using methods and tools of coordinated planning of actions in groups of robotic devices performing common tasks. Furthermore, for successful using of such systems, their functionality should allow for adjustment of formed execution plan in changing environment, including respond ability to un-foreseen situations through relocating subtasks between individual devices of group. In case of changes in the environment, their functionality must allow for adjustments of the executable plan.

According to the authors, distributed planning via interactions between UAVs has important advantages over centralized planning. Here is just a small number of these advantages:

- faster reaction to external changes than in centralized planning systems;

- simple change of execution plan (inclusion/exclusion of UAVs, replacement of faulty UAV);

- use of various models and types of UAVs.

Distributed multi-agent planning is organized through communication between individual modules on each UAV, connected to the peer-to-peer network. These modules are implemented on the base of single-board computers (such as Raspberry PI, Arduino). The developed system allows for planning and correcting actions of the UAV group when some events are triggered through interaction of individual computing modules. Therefore, tasks are solved regardless of the number of used UAVs and taking into account the amount of criteria introduced into the model which influence the overall performance.

The tasks executed using drones, which are most demanded by the market, include:

- fire and rescue operations;

- area monitoring and inspection for protection and control;

- evaluation of vegetation indexes (such as NDVI) for agriculture;

- photo and video shooting of moving objects in the spheres of leisure, tourism, entertainment.

When you create a new hardware systems designed to solve problems of group control, the most expensive stage is software development [12]. Versatility of proposed methods and their independence from the context of the problem will reduce the time for deployment of new hardware systems and reduce costs of their creating by minimizing development time of software solutions. Thus, software and hardware complexes of the new generation are composed of various models and types of UAVs capable to interact and work together and may be designed and implemented in the shortest time. Multi-agent scheduling technology is applicable to a wide variety of practical tasks in various areas of economy and industry including UAVs group management in real time. 


\section{ACKNOWLEDGMENTS}

This work was supported by Russian Foundation for Basic Research, project number 16-0100759.

\section{REFERENCES}

[1] Michael, L., Pinedo scheduling: theory, algorithms, and system, Springer, New York, p. $673,2008$.

[2] Vos, S., Meta-heuristics: the state of the art in local search for planning and scheduling, A. Nareyek (Ed.). Springer-Verlag, Berlin, pp. 1-23, 2001.

[3] Binitha, S. \& Sathya, S.S., A survey of bio inspired optimization algorithms. International Journal of Soft Computing and Engineering (IJSCE), 2(2), pp. 137-151, ISSN: 2231-2307, 2012.

[4] Rzevski, G. \& Skobelev, P., Managing complexity. WIT Press, Boston, 2014.

[5] Skobelev, P., Multi-agent systems for real time adaptive resource management. In Industrial agents: emerging applications of software agents in industry. Paulo Leitão, Stamatis Karnouskos (Ed.). Elsevier, Amsterdam, pp. 207-230, 2015.

[6] Santamaria, E., Segor, F., Tchouchenkov, I. \& Schoenbein, R., Rapid aerial mapping with multiple heterogeneous unmanned vehicles. International Journal on Advances in Systems and Measurements, 6(3-4), pp. 384-393, 2013.

[7] Di Franco, C. \& Buttazzo. G., Energy-aware coverage path planning of UAVs. Autonomous robot systems and competitions (ICARSC), 2015 IEEE International Conference, pp. 111-117, 2015.

[8] Kamrani, F., Using on-line simulation in UAV path planning. Licentiate Thesis in Electronics and Computer Systems, KTH, Stockholm, Sweden, 2007.

[9] Ergezer, H. \& Leblebicioğlu, K., 3D path planning for multiple UAVs for maximum information collection. Journal of Intelligent \& Robotic Systems, 73(1-4), pp. 737-762, 2014.

[10] Baxter, J.W., Horn, G.S. \& Leivers, D.P., Fly-by-agent: controlling a pool of uavs via a multi-agent system. The 27th SGAI International Conference on Artificial Intelligence, 21(3), pp. 232-237, 2008.

[11] Koo, T.J. \& Shahruz, S.M., Formation of a group of unmanned aerial vehicles (UAVs). American Control Conference (ACC), 2001, pp. 69-74, 2001.

[12] Austin, R., Unmanned aircraft systems UAVs design, development and deployment, 1st ed. Wiley Aerospace Series, United Kingdom, pp. 221-226, 2010. 